\title{
Motion Corrected 3D Whole-Heart Vessel Wall Imaging
}

\author{
Gastao J Lima da Cruz ${ }^{1 *}$, David Atkinson², Markus Henningsson, Rene M Botnar ${ }^{1}$, Claudia Prieto ${ }^{1}$ \\ From 19th Annual SCMR Scientific Sessions \\ Los Angeles, CA, USA. 27-30 January 2016
}

\section{Background}

Coronary atherosclerosis is not necessarily stenotic, due to outward remodeling of the vessel wall. Plaque burden correlates with risk of coronary disease (Kubo et al, J Am Coll Cardiol 2007) and direct visualization is desired. A 3D flow independent approach for vessel wall imaging was proposed recently (Andia et al, MRM 2013), based on subtraction of data with (T2prep $(+))$ and without (T2prep(-)) a T2-preparation prepulse. However, T2prep(+) and T2prep(-) data are only accepted when both are within the acquisition window given by the diaphragmatic navigator, leading to increased scan times. Here, we propose to accelerate the acquisition by performing respiratory motion correction on T2prep(-) and T2prep(+) with $100 \%$ scan efficiency. This is achieved with a beat-to-beat translational correction combined with a bin-to-bin non-rigid correction.

\section{Methods}

Data are acquired using an interleaved scanning framework (Henningsson et al, MRM 2014). T2prep(-) and $\mathrm{T} 2 \mathrm{prep}(+)$ are acquired interleaved every other heartbeat and preceded by a $2 \mathrm{D}$ coronal low resolution image navigator (iNAV) (Figure 1a). Translational foot-head (FH) and right-left (RL) motion is estimated from the iNAV and used to bin data into $\mathrm{FH}$ respiratory motion states. Within each bin, 2D translational correction is applied directly in k-space (Figure 1b). Bins are reconstructed

\section{a) Aquisition}

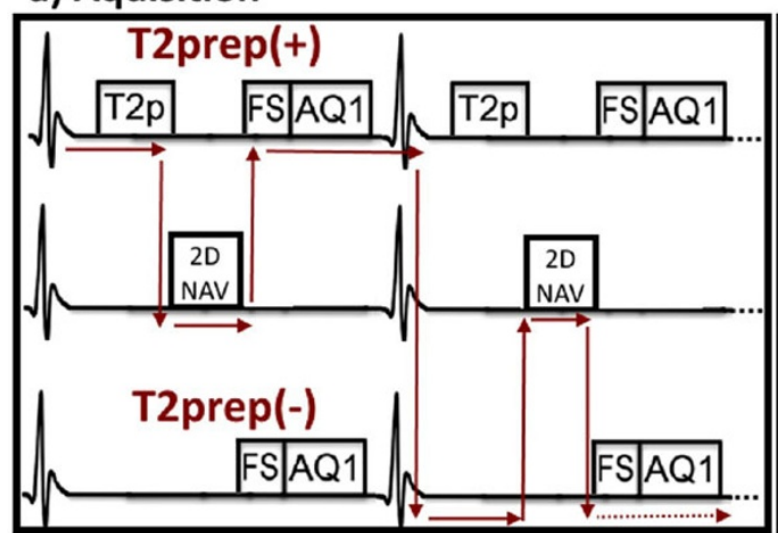

Figure 1 Proposed interleaved scanning framework: a) T2prep(+) and T2prep(-) data acquisitions are interleaved with a low-resolution 2D image navigator (iNAV). These three sequences are setup as independent sequences. b) Foot-head (FH) motion extracted from the iNAV is used to bin data. FH and right-left (RL) motion is corrected within each bin using the iNAV motion estimation. Reconstructed bins from highresolution data sets are registered to extract non-rigid motion, which is incorporated directly in the final reconstruction.

\section{b) Reconstruction}

${ }^{1}$ King's College London, London, United Kingdom

Full list of author information is available at the end of the article

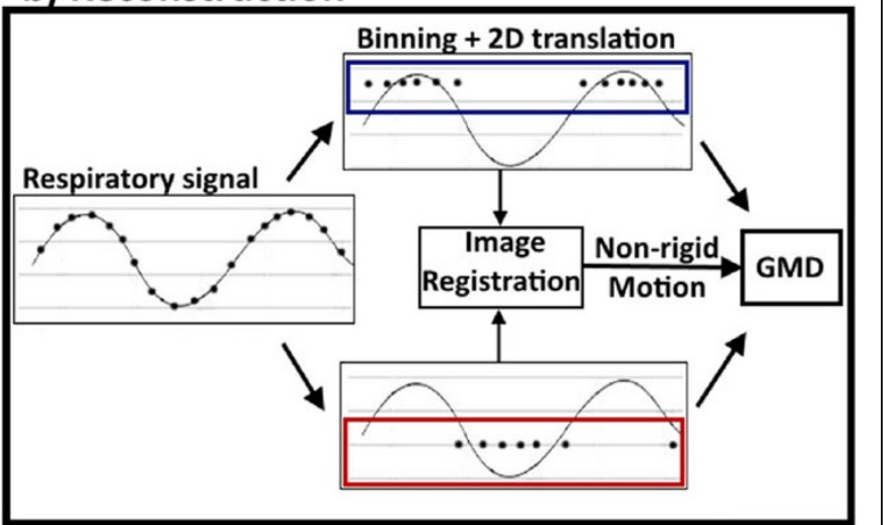

(c) 2016 Lima da Cruz et al. This is an Open Access article distributed under the terms of the Creative Commons Attribution License (http://creativecommons.org/licenses/by/4.0), which permits unrestricted use, distribution, and reproduction in any medium, provided the original work is properly cited. The Creative Commons Public Domain Dedication waiver (http://creativecommons.org/ publicdomain/zero/1.0/) applies to the data made available in this article, unless otherwise stated. 


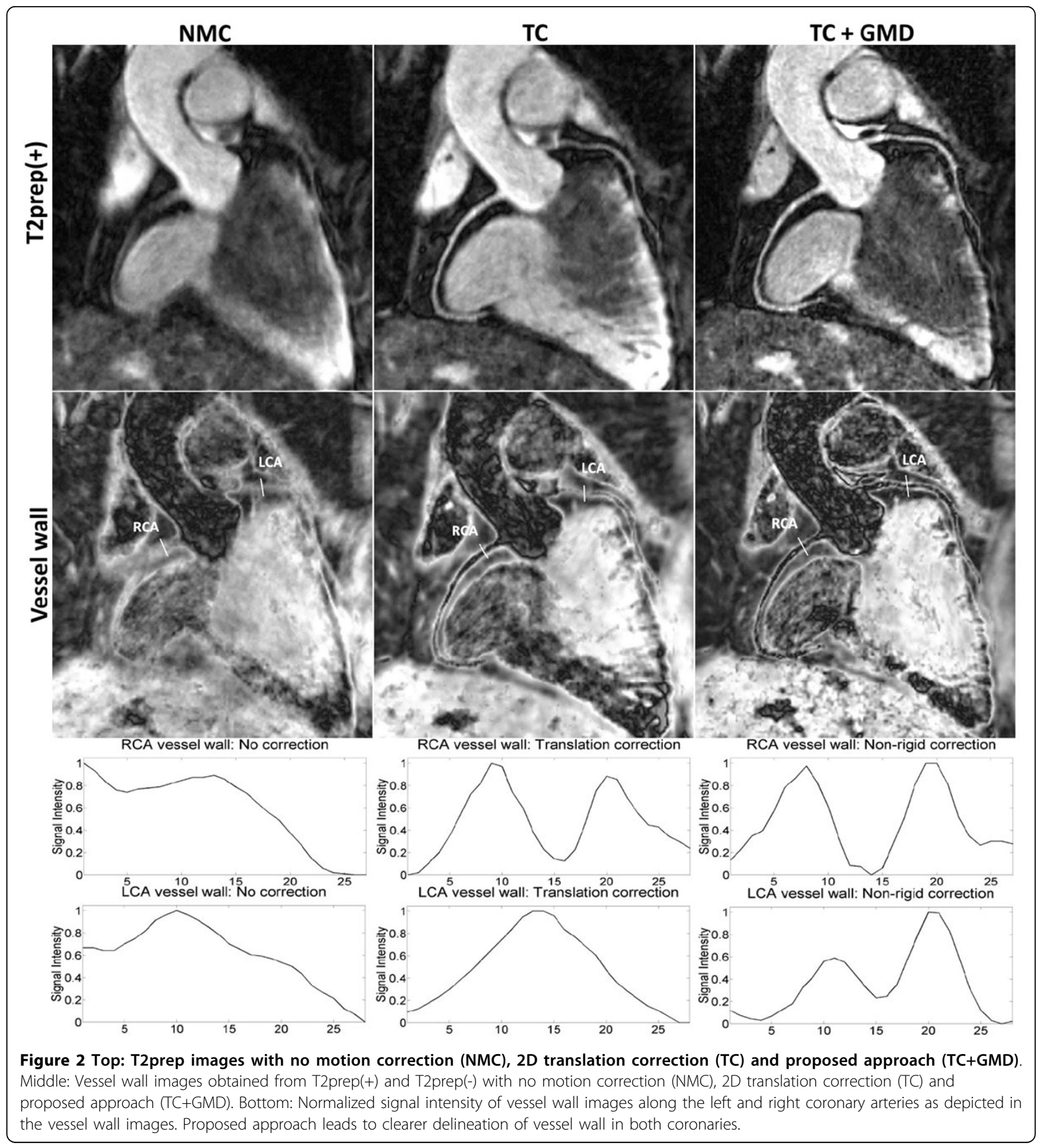

with parallel imaging and non-rigid motion is subsequently estimated via image registration. Non-rigid motion between bins is corrected using the General Matrix Description (GMD) (Batchelor et al, MRM 2005).

Eight healthy volunteers were scanned under freebreathing on a $1.5 \mathrm{~T}$ Philips scanner using a 32-channel coil. Image data was acquired with an ECG-triggered 3D balanced SSFP: coronal slices, flip angle $=70^{\circ}, \mathrm{TR} / \mathrm{TE}=$
$4.6 / 2.3 \mathrm{~ms}$, FOV $=300 \times 300 \times 100 \mathrm{~mm}^{3}$, resolution $=$ $1 \times 1 \times 2 \mathrm{~mm}^{3}$. iNAV data was acquired with a $2 \mathrm{D}$ Golden radial spoiled gradient echo: coronal slice, flip angle $=5^{\circ}, \mathrm{TR} / \mathrm{TE}=2.4 / 1.1 \mathrm{~ms}, \mathrm{FOV}=300 \times 300 \mathrm{~mm}^{2}$, resolution $=4 \times 4 \mathrm{~mm}^{2}$. Data was reconstructed with no motion correction (NMC), 2D beat-to-beat translation correction (TC) only and with the proposed approach (TC+GMD). 


\section{Results}

Reformatted T2prep(+), vessel wall images and intensity profiles along the left and right coronary arteries (LCA and RCA) are shown in Figure 2. Coronary vessels are not visible in NMC due to respiratory motion. A significant improvement is obtained in TC: RCA vessel wall becomes visible, but some residual blurring remains in the LCA. TC+GMD removes further motion artifacts leading to clearer delineation of the vessel wall in both coronary arteries. All volunteers showed visual vessel wall improvement after TC+GMD.

\section{Conclusions}

Good quality vessel wall imaging is achieved with the proposed approach with $100 \%$ scan efficiency. The proposed motion correction shows visible improvements over translational correction only, leading to a better delineation of the coronary vessel wall. Future work will focus on additional acceleration using compressed sensing.

\section{Authors' details}

'King's College London, London, United Kingdom. ${ }^{2}$ University College

London, London, United Kingdom.

Published: 27 January 2016

doi:10.1186/1532-429X-18-S1-P323

Cite this article as: Lima da Cruz et al:: Motion Corrected 3D WholeHeart Vessel Wall Imaging. Journal of Cardiovascular Magnetic Resonance 2016 18(Suppl 1):P323.

Submit your next manuscript to BioMed Central and take full advantage of:

- Convenient online submission

- Thorough peer review

- No space constraints or color figure charges

- Immediate publication on acceptance

- Inclusion in PubMed, CAS, Scopus and Google Scholar

- Research which is freely available for redistribution

Submit your manuscript at www.biomedcentral.com/submit 